\title{
Supplemental Intraoperative Oxygen and Long-term Mortality
}

Jiang Q, Kurz A, Zhang X et al. Anesthesiology 2021; 134:709-721

$\mathrm{D}$ ie Frage nach der besseren an der Narkosemaschine eingestellten intraoperativen Sauerstoffkonzentration führte zu einigen Publikationen in den letzten zwei Jahrzenten. Der ursprüngliche Gedanke war, dass eine erhöhte intraoperative Sauerstoffkonzentration von 80 Prozent zu einer erhöhten Gewebesauerstoffkonzentration führt. Diese wird von den Neutrophilen zum „oxidative killing" von Bakterien verwendet und führt somit zu einer geringeren chirurgischen Infektionsrate.

Waren anfängliche Arbeiten noch sehr vielversprechend, relativierten spätere Untersuchungen den positiven Effekt von höheren Sauerstoffkonzentrationen. Eine kürzlich veröffentlichte Metaanalyse kam zu dem Ergebnis, dass es im besten Fall nur zu einer geringen Reduktion der chirurgischen Infektionen kommt.

Auch die PROXI-Studie fand keinen Unterschied zwischen 30 vs. $80 \% \mathrm{FiO}_{2}$ in der chirurgischen Infektionsrate. Sie ließ aber damit aufhorchen, dass die Zwei-Jahres-Mortalität bei höheren Sauerstoffkonzentrationen signifikant anstieg. Dieser Effekt war bei Patient*innen nach onkologischen Eingriffen am stärksten ausgeprägt. Mögliche Erklärungsversuche reichten von Hyperoxie-induzierter Neovaskularisation oder erhöhter Erythropoietin-Produktion bis hin zu einer DANNSchädigung durch die Bildung von freien Sauerstoffradikalen.

Die Autoren dieser vorliegenden Studie versuchten der Frage nach dem Langzeit-Outcome von Patient*innen mit entweder 30 oder 80 Prozent intraoperativem Sauerstoff nachzugehen.

\section{WAS WIR BEREITS ZUM THEMA WISSEN}

- Der klinische Benefit hinsichtlich verringerter chirurgischer Infektionen bei höheren intraoperativen Sauerstoffkonzentrationen ist, wenn überhaupt, nur sehr gering.

- Höhere intraoperative Sauerstoffkonzentrationen stehen im Verdacht, speziell bei onkologischen Eingriffen die Langzeit-Mortalität zu erhöhen.

Bei der vorliegenden Studie handelt es sich um eine Posthoc-Analyse einer groß angelegten Studie aus den Jahren 2013 bis 2016 (A. Kurz et al. BJA 2018; 120:117-126). In dieser Studie wurden 4.088 Patient*innen entweder mit 30 oder mit $80 \%$ Sauerstoff intraoperativ oxygeniert. Alle Patient*innen wurden einem kolorektalen Eingriff unterzogen - mit einem großen Anteil an onkologischen Operationen.

Die Eingriffe dauerten mindestens zwei Stunden. Für die Ein- bzw. Ausleitung wurde $100 \%$ Sauerstoff verwendet. Als untere Sauerstoffsättigungsgrenze wurden $95 \%$ definiert, sodass es Patient*innen gab, die in der 30-\%-Gruppe auch höhere Sauerstoffkonzentrationen benötigten.
Das mittlere Alter der Patient*innen betrug 54 bzw. 53 Jahre. Demographische, Baseline- und Eingriffs-bezogene Variablen waren gut ausbalanciert und zeigten keine wesentlichen Unterschiede. Die Overall-Mortalität lag bei $13,8 \%$ nach einer medianen Follow-up-Periode von 3,2 Jahren.

Das Ergebnis war eindeutig: Höhere intraoperative Sauerstoffkonzentrationen erhöhen die postoperative Langzeit-Mortalität nicht.

\begin{tabular}{|l|c|c|c|c|c|}
\hline & $\mathrm{N}$ & $\begin{array}{c}2-y r \\
\text { survival } \%\end{array}$ & $\begin{array}{c}5-y r \\
\text { survival } \%\end{array}$ & $\begin{array}{c}\text { Hazard } \\
\text { Ratio }\end{array}$ & $\begin{array}{c}\mathrm{p}- \\
\text { value }\end{array}$ \\
\hline $30 \% \mathrm{FiO}_{2}$ & 1718 & $90 \%$ & $80 \%$ & Ref $=1$ & 0.493 \\
\hline $80 \% \mathrm{FiO}_{2}$ & 1753 & $91 \%$ & $80 \%$ & $\begin{array}{c}0,94 \\
(0,78- \\
1,13)\end{array}$ & \\
\hline
\end{tabular}

Auch ein kolorektaler Eingriff aufgrund einer Malignität war nicht mit einer höheren Langzeit-Mortalität durch eine erhöhte Sauerstoffkonzentration assoziiert. Weiters hatten weder die Art des Eingriffes (laparoskopisch vs. offen), Alter, BMI, Rauchen, ASA-Klassifikation noch die primären Diagnosen einen Einfluss auf das Outcome.

Die Studie schloss aus diesen Resultaten, dass ein höherer intraoperativer $\mathrm{FiO}_{2}$ in der kolorektalen Chirurgie nicht zu einer erhöhten postoperativen Langzeit-Mortalität führt. Auch bei Krebspatient*innen können höhere $\mathrm{FiO}_{2}$-Werte sicher eingesetzt werden, wenn solche benötigt werden.

FAZIT für die Praxis: Diese Studie ist in meinen Augen ein wichtiger Beitrag in der Erforschung der idealen intraoperativen Sauerstoffkonzentration für unsere Patient*innen. Zunehmend wird klar, dass höhere Sauerstoffkonzentrationen keinen positiven Einfluss auf die postoperativen Wundinfektionen und/oder eine verbesserte Anastomosenheilung haben. Auf der anderen Seite: Sollten einmal höhere $\mathrm{FiO}_{2}$-Werte benötigt werden, um eine adäquate Oxygenierung zu gewährleisten, haben diese nicht den vermuteten schlechten Einfluss auf die Krebsprognose unserer Patient*innen.

Das Fazit für unsere tägliche Arbeit lässt sich nach aktuellem publiziertem Wissen somit so darlegen: Eine intraoperative $\mathrm{FiO}_{2}$-Konzentration von 30 \% sollte aus Sicherheitsgründen als Mindeststandard angesehen werden. Höhere Sauerstoffkonzentrationen bis 80 \% können aber ohne Gefahr auf eine schlechtere Langzeitprognose zur Aufrechterhaltung einer adäquaten Oxygenierung nach Optimierung der Beatmungsparameter eingestellt werden. 